\title{
Actitudes frente al alcohol en adolescentes con propensión al consumo
}

\author{
Attitudes towards alcohol in adolescents with a propensity to consume
}

Recibido el 01 de agosto del 2017, aceptado el 11 setiembre del 2017

Horacio Cortese, Ricardo

ricardocortese@yahoo.com.ar

Facultad de Psicología y Relaciones Humanas, Universidad Abierta Interamericana - Argentina
Para referenciar este artículo: Horacio, R. (2017). Actitudes frente al alcohol en adolescentes con propensión al consumo. ConCiencia $E P G, 2(2), 36-45$.

\section{Resumen}

$\mathrm{E}$ ste estudio describió las características asociadas al consumo de alcohol y relacionó la impulsividad con las actitudes frente al alcohol en un grupo de adolescentes del Gran Buenos Aires. Además, se puso en práctica una intervención grupal. Se realizó un diseño longitudinal, de alcance descriptivo/correlacional y se trabajó con una muestra no probabilística conformada por 71 participantes de ambos sexos que cursaban el nivel secundario. Las medidas de evaluación empleadas fueron Ficha de Datos Generales, el Inventario Situacional de Consumo de Alcohol y la Escala de Impulsividad de Barratt y se confeccionó un cuestionario ad-hoc para evaluar el desempeño en el grupo de reflexión. Los resultados evidenciaron que los sujetos tuvieron sus primeras experiencias con el alcohol a partir de los 12 años de edad, con una frecuencia semanal y se encontró una asociación positiva entre la impulsividad y las actitudes frente al consumo de alcohol en quienes mostraron mayor carga emocional como actitud hacia el alcohol. Por último, se hallaron cambios significativos en las expectativas, comprensión del alcohol y la contención de los coordinadores al finalizar el grupo de reflexión.

Palabras clave: consumo de alcohol, adolescencia, impulsividad, grupo de reflexión.

\section{Summary}

his study seeked to describe the characteristics associated to alcohol consumption and link impulsiveness with the attitudes towards alcohol in a group of teenagers living in Gran Buenos Aires. Also, a group intervention was put into practice.

A longitudinal, descriptive/correlational investigation has been made and had a non-probabilistic sample conformed by 71 participants, high school level still in course. Evaluation measures used were: 
General Data Record Survey; Situational Inventory of Alcohol consumption, Scale of Impulsivity by Barratt and an ad-hoc questionnaire was madeup in order to evaluate the reflection group's performance. The results showed that subjects had their early experiences with alcohol starting by the age of 12 with a weekly periodicity, and also a positive association between impulsivity and attitudes towards alcohol consumption was found in whom showed greater emotional charge as an attitude towards alcohol consumption. At last, significant changes were found among expectations, alcohol understanding and the contention the coordinators had by the end of the reflection group.

Key words: alcohol consumption, adolescence, impulsivity, discussion group.

\section{Introducción}

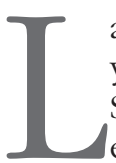
a Organización Mundial de la Salud [OMS] y la Organización Panamericana de la Salud (2014) mencionan que el consumo excesivo de alcohol es un problema de gran importancia, debido a que produce graves consecuencias sobre la salud y ocupa el tercer lugar entre los factores de riesgo de morbilidad, causa 3.3 millones de muertes cada año a nivel mundial, puede conducir a la dependencia y aumentar el riesgo de desarrollar múltiples enfermedades.

En la actualidad, el consumo de alcohol es considerado un fenómeno social que constituye un grave problema de salud pública que aumentó principalmente en la población juvenil, ya que se ha convertido en una práctica frecuente entre los adolescentes, quienes desde muy temprana edad comienzan a consumir alcohol, ya sea por curiosidad, refugio social, necesidad de adaptación o aceptación en un grupo social (Perinat et al., 2003).

En la Argentina, pese a que existen prohibiciones de venta a los menores de edad, la oferta, obtención y consumo son prácticas frecuentes; es así como los adolescentes perciben el alcohol como una bebida socialmente aceptada, según lo cual desarrollan creencias sobre su consumo (Gómez,
Luque, Ceballos, Capellino \& Bittar, 2008). De este modo, los jóvenes tienen que aprender a convivir con ello, tomando decisiones sobre su consumo responsable o abstinencia al mismo.

Cabe mencionar, que la adolescencia es considerada una etapa del ciclo vital especialmente vulnerable a la iniciación en cuanto al consumo de alcohol, ya que muchos jóvenes no se muestran preocupados por la prevención de enfermedades en el futuro, sino que se plantean sobre todo vivir el presente (Magaña, 2003). Sin embargo, son numerosos y graves los efectos del consumo de alcohol en la vida de los jóvenes pudiendo afectar su salud física y psicológica.

Asimismo, interfiere en otros aspectos puntuales del adolescente como en el rendimiento académico o el interés a realizar conductas de riesgo, como, por ejemplo, mantener contacto sexual irresponsable, conductas violentas y/o delictivas, aumentar la probabilidad de accidentes de tránsito, entre otras (Martínez, Salazar, Pedroza, Ruiz \& Ayala, 2007; Cortés, Espejo \& Giménez, 2008).

Múltiples estudios revelan que la dificultad para controlar los impulsos se encuentra relacionada con distintos comportamientos de gran impacto social, como es el abuso de sustancias (Celma, 2010). Este hecho podría observarse en los adolescentes cuando en el acto de "beber", no realizan una previa elaboración acerca de las consecuencias negativas que puede llevar el inicio de la ingesta de alcohol.

De esta manera, puede pensarse que la impulsividad, entendida en este estudio como la predisposición que tiene una persona hacia reacciones imprevistas ante estímulos, internos o externos, sin evaluar las consecuencias negativas que pueden tener tanto para uno mismo como para los demás (Barratt, 1994), interviene en el inicio y mantenimiento del consumo de alcohol en la adolescencia.

Tomando los aportes de Barrat (Figueroa, 2013; Squillace et al., 2011; Morales, 2007) existen 
tres dimensiones en la que puede evaluarse la impulsividad; éstas son: a) cognitiva, relacionada con la forma rápida en que el sujeto toma decisiones; b) motora, en la que actúa sin pensar, dejándose llevar por el ímpetu del momento, y c) no planificada, referida a la falta de previsión que tiene el sujeto hacia el futuro.

Esta concepción coincide con la de la neuropsicología, que considera que la impulsividad es la incapacidad de abstenerse a realizar un comportamiento que acarrea consecuencias negativas, en el que se privilegia lo inmediato por sobre la demora de la gratificación (Celma \& Abella, 2012).

De este modo, se infiere que la exposición continua del cerebro al alcohol hace que éste se vuelva más sensible a la gratificación inmediata que causa la mencionada sustancia psicoactiva sobre el organismo de los jóvenes.

Frente a este panorama es necesario preguntarse cómo piensan y se sienten los adolescentes respecto del consumo de alcohol y sus consecuencias. De acuerdo con esto, Costa y González (2008) mencionan que se ponen en juego aspectos cognitivos - tanto ideas y creencias asociadas al uso y abuso de alcohol, como el reconocimiento del límite- y aspectos afectivos motivación, estado de ánimo y relaciones sociales.

Cicua, Méndez y Muñoz (2008) estudiaron los factores asociados al consumo de alcohol en adolescentes de entre 12 y 17 años de edad, que residían en Bogotá. Para ello, evaluaron dos categorías: a) características de consumo: edad de inicio, frecuencia, tipo de bebida, cantidad y el lugar en donde el adolescente ha consumido alcohol; b) factores asociados: mediante las dimensiones del Inventario Situacional de Consumo de Alcohol [ISCA], situaciones personales y situaciones con otros. Los resultados evidenciaron una media de inicio de consumo a los 12 años, una frecuencia de consumo semanal, el lugar con mayor frecuencia en donde consumen alcohol fue la casa de un amigo, el tipo de bebida señalada de más consumo fue la cerveza y detectaron que el consumo era mayor en hombres que en mujeres. Respecto de los factores asociados, evidenciaron que las situaciones personales se establecían como factores de mayor riesgo para los adolescentes, que aquéllas que implicaban una interacción con otros.

Otra cuestión importante es que el consumo grupal de alcohol conforma una gran parte de la cultura adolescente e implica una concepción específica del espacio y del tiempo, un espacio común y compartido, construido por los jóvenes a través de la interacción (Pons \& Buelga, 2011).

Por esta razón, es necesario que desde el campo de la salud mental se propongan intervenciones grupales para la prevención primaria, abordando las causas del problema y no sólo las manifestaciones del mismo. Ellas se pueden llevar a cabo, si se toma en cuenta que la prevención es un intento científico para conocer los factores asociados a un problema social e intervenir desde ellos.

En función de lo expuesto, este estudio buscó describir las características asociadas al consumo de alcohol y relacionar la impulsividad (cognitiva, motora y no planeada) con las actitudes frente al alcohol en un grupo de adolescentes del Gran Buenos Aires, Argentina.

A la vez, con el fin de realizar una contribución para la prevención primaria, se puso en práctica una intervención grupal en la que adolescentes de educación media trabajaron en grupos de reflexión y se evaluaron los cambios al inicio y al finalizar el grupo, acerca de las actitudes frente al alcohol, como así también, evaluar el nivel de impulsividad.

En relación con dichos objetivos se siguieron las siguientes hipótesis: H1. Se encontrará una relación positiva y significativa entre la impulsividad $\mathrm{y}$ actitudes frente al consumo de alcohol en adolescentes; H2. La actividad grupal aumentará la expectativa, a tener mayor comprensión acerca 
del alcohol y sus consecuencias, a ser escuchado, contenido y respaldado por sus compañeros, y por los coordinadores del grupo; y H3. La actividad grupal disminuirá la impulsividad en relación con el alcohol (al finalizar el taller).

\section{Método}

Participantes: Se seleccionó una muestra no probabilística (Hernández, Fernández, \& Baptista, 2006), conformada por 71 sujetos ( $48 \%$ varones y 53\% mujeres), de entre 14 y 18 años de edad Media $=16,06 ; \mathrm{DT}=1,51$ ) que cursaban el nivel secundario de una institución educativa privada del Gran Buenos Aires. Diseño: se realizó una investigación de enfoque cuantitativo con alcance descriptivo correlacional, en corte de tiempo longitudinal dentro de un diseño experimental (Hernández et al., 2006).

Instrumentos: Se empleó un diseño de Cicua et al. (2008) para medir las características de lugar, frecuencia, cantidad, evento del consumo y datos demográficos de los adolescentes y se administró el Inventario Situacional de Consumo de Alcohol (ISCA) (Annis, 1982), que evalúa situaciones específicas de consumo de alcohol que pueden ser identificadas en un individuo como situaciones de alto riesgo, por medio de 100 reactivos que miden ocho categorías, divididas en dos grupos. El primer grupo mide Situaciones Personales, referidas a cuestionamientos de naturaleza física o psicológica; este grupo mide cinco categorías: 1) Emociones desagradables; 2) Malestar físico; 3) Emociones agradables; 4) Probando autocontrol y 5) Urgencia por consumir. El segundo grupo mide Situaciones que involucran a terceras personas y está dividido en tres categorías: 6) Conflicto con otros; 7) Presión social y 8) Momentos agradables.

Este inventario se utilizó para medir situaciones específicas de consumo de alcohol que pueden ser identificadas en un individuo como situaciones de alto riesgo de recaída. Puede ser utilizado como una herramienta para planear un tratamiento, ya que proporciona un perfil de las áreas de mayor riesgo en un individuo. Los puntajes de las 8 sub-escalas muestran confiabilidad en bebedores-problema y validez de contenido aceptable, representando el universo de situaciones de recaída (Echeburúa, 1996; Echeverría, Oviedo \& Ayala, 1998).

Se administró también la Escala de Impulsividad de Barratt adaptada al español por Oquendo (2001), diseñada para evaluar la impulsividad por medio de 30 ítems que se agrupan en 3 sub-escalas de impulsividad: cognitiva, motora y no planeada. Cada ítem consta de 4 opciones de respuesta $(0=$ Nunca, $1=$ Ocasionalmente, $3=\mathrm{A}$ Menudo, y $4=$ Casi siempre). Desde el punto de vista clínico, posee mayor relevancia el valor cuantitativo de la puntuación total.

Finalmente se diseñó una encuesta para evaluar los resultados del Taller de Reflexión [CTR]. Se confeccionó un cuestionario ad-hoc para evaluar el desempeño de la investigación-acción realizado en el grupo de adolescentes en términos de: (1) motivación para participar en grupo, (2) satisfacción de sus expectativas en pertenecer al grupo, (3) comprensión acerca del efecto del alcohol y sus consecuencias, (4) ser escuchado, contenido y respaldado por los compañeros del grupo, (5) ser escuchado, contenido y respaldado por la coordinación del grupo, y (6) tipo de beneficio logrado en el grupo. Cada pregunta se codificó en un rango de respuesta que varía desde 1 (ninguna) a 5 (muy alta). La escala se administró en dos tiempos, al inicio y al final de la actividad grupal.

Procedimiento: En primer término, se contactó con una institución educativa privada situada en la localidad de Berzategui (Gran Buenos Aires, Argentina), en la que se pidió autorización a los directivos del nivel secundario (turno mañana y tarde) para llevar a cabo el estudio y los grupos de reflexión.

Luego se solicitó por escrito a los padres o responsables adultos de los adolescentes que quisieran participar de manera anónima y voluntaria en la actividad, que firmasen el consentimiento informado en el que se comunicaba el objetivo 
del estudio, su duración y se aclaraba que era exclusivamente para fines de investigación.

Una vez terminado el procedimiento ético, se subdividieron a los participantes que accedieron a intervenir en la actividad en cuatro grupos de trabajo y durante cuatro encuentros; con cada uno de ellos se siguió el siguiente cronograma:

Primer encuentro: consistió en presentar la actividad (temática, objetivos y actividades) que se realizaría con ellos a lo largo de un mes, en un encuentro por semana que duraría 90 minutos. Posteriormente, el equipo de coordinación pidió que completen la Ficha de datos generales, el Inventario Situacional de Consumo de Alcohol (ISCA), la Escala de Impulsividad de Barratt (BIS) y por último, el Cuestionario de actividad del taller que consistía en las siguientes preguntas:

- ¿Qué te motivó a participar en el Grupo de Reflexión?

- ¿En qué medida sientes que el grupo de reflexión te ayudará a satisfacer sus expectativas?

- ¿En qué medida sientes que el grupo de reflexión te ayudará a tener una comprensión acerca del alcohol y sus consecuencias?

- ¿En qué medida sientes que serás escuchado, contenido y respaldado por tus compañeros del grupo?

- ¿En qué medida sientes que serás escuchado, contenido y respaldado por la coordinación del grupo?

- ¿Cuánto crees que tu participación en el taller te beneficiará en otros aspectos?

Segundo encuentro: consistió en el desarrollo de la dinámica en el grupo de reflexión, por medio de talleres teóricos participativos en el que se utilizaron los siguientes recursos: métodos y enfoques activos; proceso de enseñar y aprender; compartir la información (problemas comunes); realidad integradora, compleja y reflexiva; unión de la teoría y la práctica y fuerza motriz del proceso pedagógico.

Tercer encuentro: referido a realizar acciones focalizadas, subdivididas en los siguientes ejes: formación de la identidad; representación de sí mismo; necesidad de reconocimiento y aceptación; formación de un concepto positivo de sí mismo; afloramiento de una moral autónoma; normas que emergen de las relaciones de reciprocidad y cooperación; promoción de valores y hábitos y experimentación de nuevos roles.

Cuarto encuentro: se realizaron seis actividades de dinámicas de grupo extraídas de "Promoviendo la adaptación saludable de nuestros adolescentes" de Ruiz (1998), a saber:

Dinámica de presentación: nos ponemos en lugar del otro, para favorecer la empatía.

Contacto con tacto, con el fin de ejercitar habilidades de comunicación no verbal, vencer el miedo al contacto físico y reflexionar sobre cómo el sentirse observado mediatiza la comunicación.

Evaluación proyectiva del autoconcepto, para explorar la autoimagen y los deseos de los adolescentes, separar la conducta que exhiben de su verdadera forma de ser y poder reflexionar sobre cómo influyen los demás sobre su autoconcepto y su conducta.

Como nos ven: dinámica para conocer la visión que tienen los demás de cada uno y valorar si coincide o no con la visión que cada uno tiene de sí mismo.

Caricias sin pellizcos: ejercicio que sirve para ejercitar habilidades de dar y recibir elogios realistas.

Los tres caramelos: actividad en que se busca ejercitar las habilidades de seducción hablando un minuto sobre quién es uno y por qué merece la pena ser conocido. 
Posteriormente, se realizó el cierre del taller y los participantes completaron nuevamente la BIS, el ISCA y el Cuestionario de actividad del taller con las siguientes preguntas: 1. ¿Qué te motivó a participar en el Grupo de Reflexión? ¿En qué medida sientes que el grupo de reflexión te ayudó a satisfacer sus expectativas? ¿En qué medida sientes que el grupo de reflexión te ayudó a tener una comprensión acerca del alcohol y sus consecuencias? ¿En qué medida sientes que has sido escuchado, contenido y respaldado por tus compañeros del grupo? ¿En qué medida sientes que has sido escuchado, contenido y respaldado por la coordinación del grupo? ¿En qué medida Sientes que tu participación en el taller te ha beneficiado en otros aspectos? Finalmente, se codificaron los datos en el programa IBM SPSS 22.0 (Statistical Package for Social Sciences), para realizar el análisis estadístico correspondiente.

\section{Resultados}

Primero se realizó el análisis estadístico descriptivo de las características de consumo en donde se encontró que la mayoría de los adolescentes que participaron iniciaron su consumo de alcohol entre los $12(26,8 \%), 13(25,4 \%)$ y $14(25,4 \%)$ años de edad. A su vez, la mayoría consume con una frecuencia semanal $(54,9 \%)$, la bebida preferente es el fernet $(64,8 \%)$ seguida por la cerveza $(60,6 \%)$ y la discoteca es el lugar en dónde realizan el consumo (40,9\%).

Luego, se realizó la correlación entre las Actitudes hacia el Alcohol y la Impulsividad, en dónde no se encontraron relaciones significativas entre ambas variables, aunque se encontró una relación positiva entre la Impulsividad Motora y Cognitiva con Emociones Agradables y Desagradables ( $\mathrm{p}=.015$ y .043).

Tabla 1

Relación entre las Actitudes hacia el Alcohol y la Impulsividad

\begin{tabular}{lllll}
\hline Actitudes frente al Alcohol & $\begin{array}{l}\text { F1. } \\
\text { Cognitiva }\end{array}$ & $\begin{array}{l}\text { F2. } \\
\text { Motora }\end{array}$ & $\begin{array}{l}\text { F3. } \\
\text { No Planeada }\end{array}$ & $\begin{array}{l}\text { Impulsividad } \\
\text { (total) }\end{array}$ \\
\hline 1. Malestar físico & 0,108 & 0,183 & $-0,019$ & 0,064 \\
2. Emociones Desagradables & 0,379 & 0,134 & 0,876 & 0,605 \\
&, $278^{*}$ &, $390^{* *}$ & 0,136 &, $293^{*}$ \\
3. Emociones Agradables & 0,022 & 0,001 & 0,269 & 0,015 \\
4. Probando Autocontrol &, $255^{*}$ &, $321^{* *}$ & 0,143 &, $246^{*}$ \\
& 0,036 & 0,008 & 0,245 & 0,043 \\
5. Conflicto con Otros & 0,094 &, $250^{*}$ & $-0,015$ & 0,109 \\
& 0,444 & 0,039 & 0,904 & 0,377 \\
6. Necesidad Física & 0,15 &, $268^{*}$ & $-0,021$ & 0,11 \\
7. Presión Social & 0,222 & 0,027 & 0,864 & 0,373 \\
8. Momentos Agradables & 0,212 &, $360^{* * *}$ & 0,056 & 0,23 \\
& 0,083 & 0,003 & 0,652 & 0,059 \\
Alcohol (Total) &, $283^{*}$ &, $329^{* *}$ & 0,027 & 0,214 \\
\hline
\end{tabular}


Se realzó una comparación de cada participante del taller mediante un test de $\mathrm{t}$ para muestras relacionadas. Se encontraron cambios significativos

Tabla 2

Expectativas, comprensión y contención en el taller al inicio del grupo y al finalizar

\begin{tabular}{|c|c|c|c|c|c|c|c|c|}
\hline \multirow{2}{*}{ Cuestionario* } & & \multicolumn{2}{|l|}{ Antes } & \multicolumn{2}{|c|}{ Después } & \multirow[b]{2}{*}{$t^{*}$} & \multirow[b]{2}{*}{$\mathrm{P}$} & \\
\hline & & Media & DT & Media & DT & & & \\
\hline \multicolumn{2}{|c|}{ Expectativas satisfechas } & 2,56 & 1,19 & 3,75 & 0,907 & 5,82 & \multicolumn{2}{|c|}{$<, 001$} \\
\hline \multicolumn{2}{|c|}{ Comprensión del alcohol } & 3,27 & 1,19 & 3,76 & 1,1 & 2,17 & \multicolumn{2}{|c|}{0,034} \\
\hline \multicolumn{2}{|c|}{ Contención de compañeros } & 2,74 & 1,28 & 3,15 & 1,16 & 1,96 & \multicolumn{2}{|c|}{0,055} \\
\hline \multicolumn{2}{|c|}{ Contención de coordinadores } & 2,75 & 1,26 & 4,06 & 1,02 & 6,03 & \multicolumn{2}{|c|}{$<, 001$} \\
\hline \multicolumn{9}{|c|}{$\begin{array}{l}\text { * Test de t de muestras relacionadas. } \\
* * \text { Rango } 1=\text { Ninguna a } 5=\text { Muy alta. }\end{array}$} \\
\hline \multicolumn{4}{|c|}{$\begin{array}{l}\text { Por último, se llevó a cabo una comparación de } \\
\text { cada participante del taller mediante un test de } \\
\text { t para muestras relacionadas. No se encontraron }\end{array}$} & \multicolumn{5}{|c|}{$\begin{array}{l}\text { cambios significativos en la impulsividad, aunque } \\
\text { levemente significativo en Impulsividad Cognitiva } \\
(\mathrm{p}=.05) \text {. }\end{array}$} \\
\hline \multicolumn{9}{|l|}{ Tabla 3} \\
\hline \multirow[b]{2}{*}{ Cuestionario* } & \multicolumn{3}{|l|}{ Antes } & \multicolumn{3}{|l|}{ Después } & \multirow[b]{2}{*}{$t^{*}$} & \multirow[b]{2}{*}{$\mathrm{P}$} \\
\hline & Media & DT & & Media & DT & & & \\
\hline F1. Cognitiva & 12,33 & 3,57 & & 11,3 & 3,2 & & 2 & 0,05 \\
\hline F2. Motora & 14,56 & 5,72 & & 14,79 & 6,08 & & 0,33 & 0,736 \\
\hline F3. No Planeada & 13,72 & 4,78 & & 14,42 & 4,3 & & 1,01 & 0,314 \\
\hline Impulsividad (total) & 40,61 & 10,6 & & 40,51 & 9,9 & & 0,09 & 0,925 \\
\hline
\end{tabular}

* Test de $t$ de muestras relacionadas.

\section{Discusión}

Los resultados obtenidos evidenciaron que la mayor parte de adolescentes que participaron en este estudio, inician el consumo de bebidas alcohólicas entre los 12 y 14 años de edad y presentan un patrón de consumo semanal, siendo la discoteca el lugar de preferencia para llevar a cabo la ingesta. en las expectativas $(\mathrm{p}=.001)$, comprensión del alcohol $(\mathrm{p}=.03)$ y la contención de los coordinadores del taller $(\mathrm{p}<.001)$ al finalizar el taller. 
mayor medida con casi todas las actitudes hacia el alcohol menos con malestar físico; mientras que la dimensión cognitiva de la impulsividad, sólo se vinculó positivamente con tres actitudes (emociones agradables y desagradables y presión social). Por último, la dimensión planeada no presentó ninguna relación estadísticamente significativa con las actitudes frente al alcohol (Tabla 1).

Con respecto a los talleres realizados en el grupo de reflexión, los resultados mostraron un aumento en las expectativas que poseían los adolescentes, la comprensión acerca del consumo de alcohol y la contención por parte de los coordinadores al finalizar la actividad (Tabla 2).

Finalmente, no se encontraron diferencias significativas entre los niveles de Impulsividad de los adolescentes que presentaron al inicio y al final de los talleres, sólo se evidenció una leve disminución a nivel cognitivo al finalizar el grupo (Ver Tabla 3).

\section{Conclusión}

Este trabajo buscó conocer los aspectos personales y grupales que intervienen en el consumo de alcohol de un grupo de adolescentes de la localidad de Berazategui, Gran Buenos Aires, a través del estudio de la impulsividad, las actitudes hacia la bebida y la puesta en marcha de grupos de reflexión.

En primer lugar, se indagaron las características descriptivas del fenómeno y se encontró que los participantes del estudio informaron haber tenido experiencias con el consumo de bebidas alcohólicas, en especial fernet y cerveza, a partir de los 12 años de edad, con una frecuencia semanal, siendo la discoteca el lugar de preferencia en dónde ejercían la acción.

Estos datos, avalan que la ingesta de alcohol en adolescentes presenta un patrón de desarrollo temprano (Morales, 2008) y su abuso ocurre en lugares recreativos, tal como lo es la discoteca, en donde se ponen en juego creencias de alivio, facilitadoras o permisivas, mediante las que los jóvenes minimizan los riesgos asociados al alcohol (Gómez et al., 2008).

En segundo lugar, se relacionó la impulsividad (cognitiva, motora y no planeada) con las actitudes frente al alcohol y se planteó como hipótesis que se encontraría una asociación positiva y significativa entre la impulsividad y las actitudes frente al consumo de alcohol en adolescentes. De acuerdo a los resultados, esta relación no se confirmó excepto para quienes mostraron mayor carga emocional -agradable o desagradable- como actitud hacia el alcohol, que tendieron a mostrar mayor impulsividad -motora y cognitiva-.

De esta manera, puede pensarse que las emociones que los jóvenes depositan en la bebida se relacionan con la forma rápida en tomar la decisión de beber dejándose llevar por el momento, priorizando la gratificación inmediata a corto plazo. Esto coincide con el estudio de Costa y González (2008), en el que indicaron que los aspectos afectivos y cognitivos son un factor de riesgo en el patrón de consumo de alcohol en la adolescencia.

Por otra parte, con el propósito de realizar un aporte preventivo hacia esta problemática, se puso en práctica una intervención grupal por medio de grupos de reflexión en la que los adolescentes trabajaron con diversas dinámicas. En éstas se evaluaron los cambios, al inicio y al finalizar el grupo, tanto en las actitudes frente al alcohol, como en el nivel de impulsividad y se hipotetizó que la actividad grupal ayudaría a satisfacer las expectativas, a tener mayor comprensión acerca del alcohol y sus consecuencias, a ser escuchados, contenidos y respaldados por sus compañeros y por los coordinadores del grupo, lo cual se confirmó. Se encontraron cambios significativos en las expectativas, comprensión del alcohol y la contención de los coordinadores al finalizar el taller.

Londoño y Valencia (2010) mencionan que el entorno físico y social provee las claves para la 
acción y actúan como señales ambientales que motivan el consumo. En consecuencia, es relevante abordar este fenómeno en el contexto escolar, mediante el desarrollo de intervenciones, como la realizada en este estudio, para que los adolescentes sean prevenidos de las consecuencias negativas que conlleva el abuso de sustancias, pudiendo manifestar sus pensamientos y sentimientos y por, sobre todo, sentirse escuchados y comprendidos por los adultos.

Por último, este estudio propuso una aproximación a la problemática del consumo de alcohol en adolescentes, por lo que se cree necesario que se abran nuevas líneas de investigación desde el campo de la salud mental para poder enriquecer las estrategias presentes en los programas de promoción y prevención de salud, ajustando las limitaciones que presentó este estudio. Dichas limitaciones fueron: el tamaño de

\section{Referencias}

Annis, H.M. (1982). Inventory of Drinking Situations. Toronto: Addiction Research Foundation of Ontario.

Barratt, E. (1994). Impulsiveness and aggression. En J. Monahan \& H. J. Steadman (Eds.), Violence and mental disorder: developments in risk assessment. (pp. 61-79). Chicago: The University Chicago Press.

Celma, J. (2010). Impulsividad y tabaco. En Info tabac, 20, 1-6.

Celma, J. \& Abella, P. (2012). Neuropsicología de la impulsividad. Actualizaciones. Barcelona: Universitat de Lleida.

Cicua, D., Méndez, M., \& Ortega, L. M. (2008). Factores en el consumo de alcohol en adolescentes. Pensamiento psicológico, 4 (11), 115-134.

Cortés, M., Espejo, B. \& Giménez, J. (2008). Aspectos cognitivos relacionados con la práctica del botellón. Psicothema, 20 (3), 396-402. la muestra fue reducido y el proceso de muestreo empleado no garantiza la representatividad de la muestra y la generalización de los resultados hallados. Además, no se ha controlado el efecto de otras variables como el género, uso de otras sustancias psicoactivas (ej. nicotina), estado psíquico actual, antecedentes familiares de consumo, entre otras, que pueden amenazar la validez interna del estudio.

En conclusión, estos resultados permiten profundizar la información disponible y plantear alternativas ajustadas a las necesidades reales de los jóvenes, pues como menciona el equipo de Cicua (2008, p. 121) "es necesario dirigir una mirada hacia el mundo adolescente, lo que implica profundizar en las características y cambios en el constante desarrollo cognitivo, social y cultural; así como el concepto y las implicaciones, del ser adolescente".

Costa, A. M. \& González, C. (2008). Definiendo el concepto abuso de alcohol desde el sentido que los dolescentes atribuyen a su práctica. $X V$ Jornadas de Investigación y Cuarto Encuentro de Investigadores en Psicología del Mercosur. Buenos Aires: Facultad de Psicología, Universidad de Buenos Aires.

Echeburúa, E. (1996). El Alcoholismo. Madrid: Aguilar.

Echeverría, L., Oviedo, P. \& Ayala, H. (1998). Inventario Situacional de Consumo de Alcohol (ISCA). En H. Ayala, G. Cárdenas, L. Echeverría, y M. Gutiérrez (Ed). Manual de autoayuda para personas con problemas en su forma de beber. México: Facultad de Psicología, UNAM.

Figueroa, M. (2013). Evaluación conductual y psicofisiológica de la impulsividady su relación con el trastorno por déficit de atención con hiperactividad (TDAH) (Tesis doctoral no publicada). Universidad de Granada, España. 
Gómez, R. Á., Luque, L. E., Ceballos, M., Capellino, M. \& Bittar, C. (2008). consumo de alcohol en adolecentes: el rol de las creencias adictivas. $X V$ Jornadas de Investigación y Cuarto Encuentro de Investigadores en Psicología del Mercosur. Buenos Aires: Facultad de Psicología, Universidad de Buenos Aires.

Hernández, R., Fernández, C. \& Baptista, P. (2006). Metodología de la investigación. México: McGraw-Hill.

Londoño, C. \& Valencia, S. (2010). Resistencia de la presión de grupo, creencias acerca del consumo y consumo de alcohol en universitarios. Anales de Psicología, 26(1), 2733.

Magaña, M. (2003). La adolescencia hoy. Anales de pediatría, 58 (2), 95-96.

Martínez, K., Salazar, M., Pedroza, F., Ruiz, G. \& Ayala, H. (2008). Resultados preliminares del programa de intervención breve para adolescentes que inician el consumo de alcohol y otras drogas. Salud mental, 31(2), 119-127.

Morales, F. (2007). El efecto de la impulsividad sobre la agresividad y sus consecuencias en el rendimiento de los adolescentes (Tesis de doctorado no publicada). Universidad de Rovira I Virgili, España.

Morales, H. (2008). Factores asociados y trayectorias del desarrollo del comportamiento antisocial durante la adolescencia: implicancias para la prevención de la violencia juvenil en América Latina. Interamerican Journal of Psychology, 42(1), 129-142.
Organización Panamericana de la Salud y Organización Mundial de la Salud (2014). Informe mundial de la OMS destaca los impactos negativos del alcohol en la salud. Recuperado de http:// www.paho.org/arg/index.php?option=com content\&view=article\&id=1317\&Itemid=226

Oquendo, M. A., Baca-Garcia, E., Graver, R., Morales, M., Montalban, V., \& Mann, J. J. (2001). Spanish adaption of the Barratt impulsiveness scale (BIS). European Journal of Psychiatry, 15, 147-155.

Perinat, A., Corral, A., Crespo, I., Doménech, E., FontMayolas, S., Lazuela, J., Larraburu, I., Martínez, G., Moncada, A., Raguz, M. \& Rodríguez-Tomé, H. (2003). Los adolescentes en el siglo XXI. Barcelona: UOC.

Pons, J. \& Buelga, S. (2011). Factores asociados al consumo juvenil de alcohol: una revisión desde una perspectiva psicosocial y ecológica. Psychosocial Intervention, 20(1), 75-94.

Ruiz, P. (1998). Promoviendo la adaptación saludable de nuestros adolescentes. Madrid: Ministerio de sanidad y consumo centro de publicaciones.

Squillace, M., Picón, J. \& Schmidt, V. (2011). El concepto de impulsividad y su ubicación en las teorías psicobiológicas de la personalidad. Revista Neuropsicología Latinoamericana, 3(1), 8-18. 